\title{
Prognostic Value of Troponin Elevation in COVID-19 Hospitalized Patients
}

\author{
Elena-Mihaela Cordeanu ${ }^{1, *}$, Nicolas Duthil ${ }^{1}$, Francois Severac ${ }^{2}$ (D) Hélène Lambach ${ }^{1}$, \\ Jonathan Tousch $^{1}$, Lucas Jambert ${ }^{3}$, Corina Mirea ${ }^{1}$, Alexandre Delatte ${ }^{4}$, Waël Younes ${ }^{5}$, \\ Anne-Sophie Frantz ${ }^{1}$, Hamid Merdji ${ }^{6}$, Valérie Schini-Kerth ${ }^{7}$ (D), Pascal Bilbault ${ }^{8}$, \\ Patrick Ohlmann ${ }^{9}$, Emmanuel Andres ${ }^{10}$ and Dominique Stephan ${ }^{1}$
}

1 Department of Hypertension, Vascular Disease and Clinical Pharmacology, Strasbourg Regional University Hospital, 67091 Strasbourg, France; nicolas.duthil@chru-strasbourg.fr (N.D.); helene.lambach@chru-strasbourg.fr (H.L.); jonathan.tousch@chru-strasbourg.fr (J.T.); corina.mirea@chru-strasbourg.fr (C.M.); anne-sophie.frantz@chru-strasbourg.fr (A.-S.F.); dominique.stephan@chru-strasbourg.fr (D.S.)

2 Division of Public Health, Methodology and Biostatistics, University Hospitals of Strasbourg, 67091 Strasbourg, France; francois.severac@chru-strasbourg.fr

3 Department of Vascular Medicine, Mulhouse Regional Hospital, 68100 Mulhouse, France; jambertlucas@gmail.com

4 Department of Cardiology, Haguenau Regional Hospital, 67500 Haguenau, France; delatte.alex@gmail.com

5 Department of Vascular Medicine, Colmar Regional Hospital, 68000 Colmar, France; wael.younes@ch-colmar.fr

6 Intensive Care and Reanimation Department, Strasbourg Regional University Hospital, 67091 Strasbourg, France; hamid.merdji@chru-strasbourg.fr

7 UMR 1260 INSERM Regenerative Nanomedecine, Faculty of Pharmacy, Strasbourg University, 67400 Illkirch, France; valerie.schini-kerth@unistra.fr

8 Emergency Department, Strasbourg Regional University Hospital, 67091 Strasbourg, France; pascal.bilbault@chru-strasbourg.fr

9 Cardiology Department, Strasbourg Regional University Hospital, 67091 Strasbourg, France; patrick.ohlmann@chru-strasbourg.fr

10 Internal Medicine Department, Strasbourg Regional University Hospital, 67091 Strasbourg, France; emmanuel.andres@chru-strasbourg.fr

* Correspondence: elena-mihaela.cordeanu@chru-strasbourg.fr; Tel.: +33-0369-551-520

Received: 10 November 2020; Accepted: 15 December 2020; Published: 17 December 2020 updates

\begin{abstract}
Background: Severe acute respiratory syndrome coronavirus 2 (SARS-CoV-2) penetrates the respiratory epithelium through angiotensin-converting enzyme-2 (ACE2) binding. Myocardial and endothelial expression of ACE2 could account for the growing body of reported evidence of myocardial injury in severe forms of Human Coronavirus Disease 2019 (COVID-19). We aimed to provide insight into the impact of troponin (hsTnI) elevation on SARS-CoV-2 outcomes in patients hospitalized for COVID-19. (2) Methods: This was a retrospective analysis of hospitalized adult patients with the SARS-CoV-2 infection admitted to a university hospital in France. The observation period ended at hospital discharge. (3) Results: During the study period, 772 adult, symptomatic COVID-19 patients were hospitalized for more than $24 \mathrm{~h}$ in our institution, of whom $375 \mathrm{had}$ a hsTnI measurement and were included in this analysis. The median age was 66 (55-74) years, and there were $67 \%$ of men. Overall, $205(55 \%)$ patients were placed under mechanical ventilation and $90(24 \%)$ died. A rise in hsTnI was noted in $34 \%$ of the cohort, whereas only three patients had acute coronary syndrome (ACS) and one case of myocarditis. Death occurred more frequently in patients with hsTnI elevation (HR 3.95, 95\% CI 2.69-5.71). In the multivariate regression model, a rise in hsTnI was independently associated with mortality (OR 3.12, 95\% CI 1.49-6.65) as well as age $\geq 65$ years old (OR 3.17, 95\% CI 1.45-7.18) and CRP $\geq 100 \mathrm{mg} / \mathrm{L}$ (OR 3.62, 95\% CI 1.12-13.98). After performing a sensitivity analysis for the missing values of hsTnI, troponin elevation remained independently and
\end{abstract}


significantly associated with death (OR 3.84, 95\% CI 1.78-8.28). (4) Conclusion: Our study showed a four-fold increased risk of death in the case of a rise in hsTnI, underlining the prognostic value of troponin assessment in the COVID-19 context.

Keywords: COVID-19; troponin; myocardial injury; SARS-CoV-2; cardiovascular; biomarker

\section{Introduction}

Human Coronavirus Disease 2019 (COVID-19), resulting from a newly described respiratory viral infection with severe acute respiratory syndrome coronavirus 2 (SARS-CoV-2), was originally identified in December 2019 in Wuhan, China, before becoming a global pandemic. Cardiovascular risk factors and underlying cardiovascular diseases were associated with worse prognosis [1]. Moreover, cardiovascular involvement in COVID-19 has been related to the viral infective mechanism through the binding of the spike envelope protein to cell membrane angiotensin-converting enzyme-2 (ACE2) [2,3]. ACE2 is physiologically implicated in balancing the deleterious effects of renin-angiotensin system activation, being highly expressed by the lungs, kidneys, gut, and brain and has also been identified in the cardiovascular system [4]. Endothelial and myocardial expression of ACE2 could account for myocardial injury, defined by a rise in troponin (Tn) associated with some severe forms of COVID-19 [5,6]. In March 2020, the American College of Cardiology suggested Tn measurement in the context of COVID-19 only if myocardial infarction was suspected [7]. Since then, myocardial injury has been described in approximately one-third of COVID-19 patients [8-13]. Recent publications have associated an elevation in cardiac and inflammatory biomarkers with infection severity and worse prognosis $[13,14]$. Whether cardiac biomarkers such as Tn could have a prognostic value in SARS-CoV-2 is still under debate, and Tn measurement is not systematically performed in infected patients. We report herein a retrospective analysis of hospitalized adult patients, in whom a high-sensitivity troponin I (hsTnI) test was performed, from a university hospital in Eastern France, one of the most affected areas in Europe during the first wave of the COVID-19 pandemic.

\section{Experimental Section}

\subsection{Study Design and Patient Selection}

We performed a retrospective analysis of electronic medical records of hospitalized COVID-19 patients admitted to the University Hospital of Strasbourg between 25 February 2020 (date of admission of the first case) and 1 April 2020. The study was approved by the Strasbourg University Hospital Ethical Committee. All patients aged more than 18 years old were selected on the basis of laboratory-confirmed COVID-19 infection by positive reverse-transcriptase polymerase chain reaction (RT-PCR) on a nasopharyngeal swab. A local RT-PCR kit was used to detect SARS-CoV-2. The observation period ended at discharge. Vital status at discharge was known for all hospitalized patients. Thus, all patients having had at least one measurement of hsTnI during hospitalization were included in the first analysis and the entire cohort in the sensitivity analysis.

\subsection{Baseline Variables}

Data concerning medical history, chronic medication, clinical presentation, laboratory findings, and low-dose pulmonary computed tomography (CT) lesions were collected. hsTnI test results were retrieved from two hospital sites with site- and sex-specific 99th percentile upper reference limits (URLs) (Hospital Site 1: Siemens Advia Centaur XP and XPT assay with URLs of $37 \mathrm{ng} / \mathrm{L}$ for women and $57 \mathrm{ng} / \mathrm{L}$ for men; Hospital Site 2: Siemens Dimension Vista assay with URLs of $54 \mathrm{ng} / \mathrm{L}$ for women and $79 \mathrm{ng} / \mathrm{L}$ for men). When several tests were performed for the same patient, maximum levels of hsTnI were recorded. Given the infectivity risk and according to guidelines, echocardiography 
was only sporadically performed, and data were colligated. Antiviral, antibiotic, and anticoagulant treatments during hospitalization were equally reported.

\subsection{Outcome Assessment}

For the purpose of this study, the observation period ended at hospital discharge, with a median length of stay of 16.5 days (interquartile range (IQR): 8-29). All patient data were collected during hospitalization. The main evaluation criterion was in-hospital death from any cause. Recourse to high-flow nasal oxygen (HFNO) therapy, noninvasive ventilation (NIV), orotracheal intubation (OTI), and occurrence of severe sepsis, arterial or venous thrombosis, and acute renal impairment were colligated. Events were abstracted from clinical charts or discharge synopsis. The evaluation criteria were adjudicated by senior physicians of the vascular medicine unit.

\subsection{Statistical Analysis}

This was a retrospective cohort study; therefore, no power calculation was performed. Continuous variables were expressed as mean \pm standard deviation $(\mathrm{SD})$ or median with interquartile range (IQR), depending on their distribution. The normality of the distribution was assessed using the Shapiro-Wilk test. Categorical variables were presented as numbers of cases (percentages/frequencies). Continuous variables were compared using the Student's $t$-test or Wilcoxon rank-sum test, for categorical variables, Fisher's exact tests were employed. In order to address potential sources of bias, clinically pertinent risk factors associated with mortality in univariate analysis were selected as candidates for the multivariate logistic regression analysis. Results were expressed as odds ratios (ORs) with $95 \%$ confidence intervals (CI). A sensitivity analysis including patients without troponin measurement was performed using multiple imputation by chained equation to handle missing values [15,16]. A value of $p<0.05$ was considered statistically significant. All analyses were performed using R software version 3.2.2 (R Foundation for Statistical Computing, Vienna, Austria. URL https://www.R-project.org/).

\section{Results}

\subsection{Patients Characteristics at Baseline}

A total of 943 COVID-19 patients were admitted to the University Hospital of Strasbourg from 25 February 2020 to 1 April 2020, of whom 375 (67\% of males, mean age of $66 \pm 14.4$ ranging from 21 to 93 years) were included in this analysis after exclusion of patients hospitalized for less than $24 \mathrm{~h}$ $(n=145)$, minors $(n=14)$, patients hospitalized for other medical reasons and incidentally found positive for SARS-CoV-2 PCR $(n=12)$, and patients without a hsTnI test $(n=397)$ (Figure 1$)$.

The remaining population was divided into two subgroups based on hsTnI elevation, namely "elevated hsTnI" $(n=126)$ and "normal hsTnI" $(n=249)$. A comprehensive, comparative description of baseline characteristics and in-hospital outcomes for patients having an elevated hsTnI versus normal hsTnI test is presented in Table 1. A rise in troponin was associated with higher age and comorbid conditions such as pre-existing hypertension, diabetes, dyslipidemia, chronic heart failure, and chronic kidney disease. In-hospital treatment (antiviral, antibiotics, and anticoagulation) did not differ between groups (Table 1). 
943 patients diagnosed with SARS-CoV-2 infection from Feb 25th, 2020 to April 1st, 2020

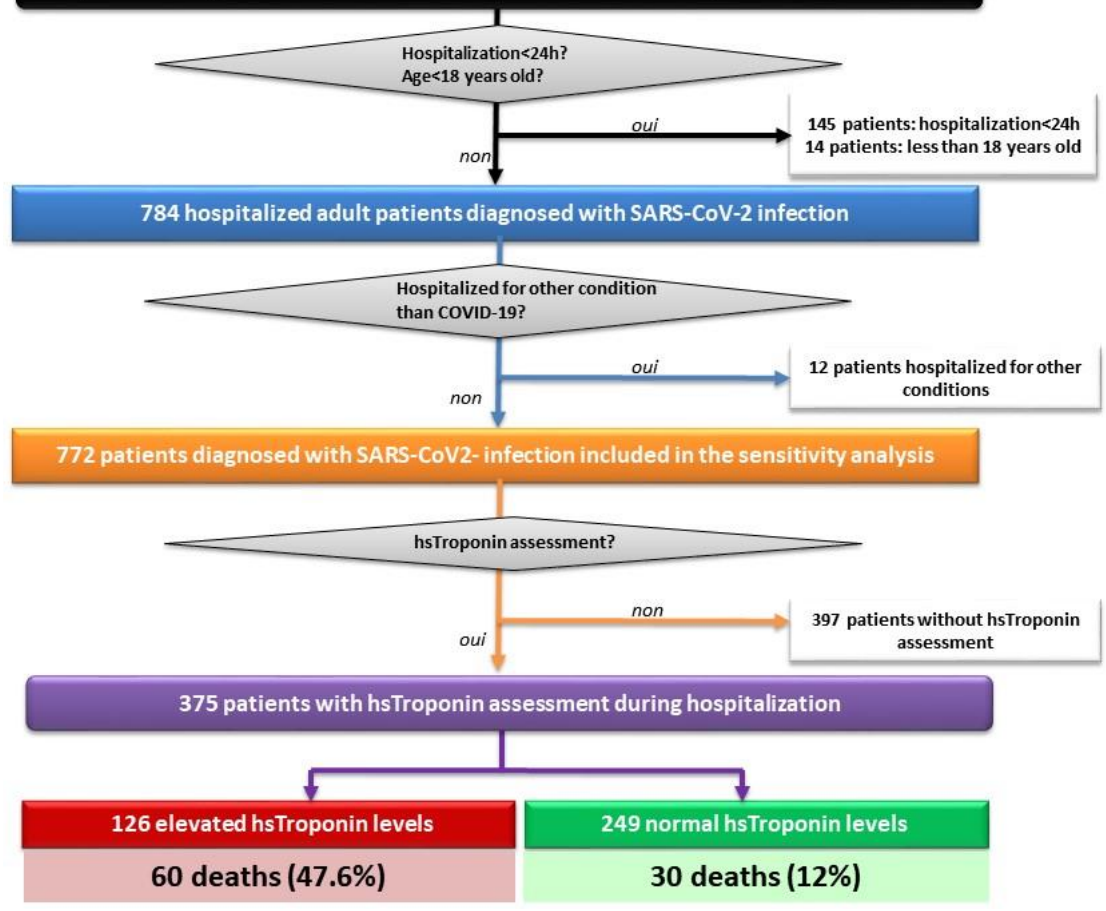

Figure 1. Study flowchart showing patient selection. COVID-19: Human Coronavirus Disease 2019; Feb: February; h: hours; hs: high-sensitivity; SARS-CoV-2: severe acute respiratory syndrome coronavirus 2.

Table 1. Baseline characteristics and in-hospital outcomes.

\begin{tabular}{|c|c|c|c|c|c|}
\hline & $\begin{array}{l}\text { Overall Cohort } \\
\text { N (\%)/M(IQR) }\end{array}$ & $\begin{array}{l}\text { Elevated hsTnI } \\
\text { N (\%)/M(IQR) }\end{array}$ & $\begin{array}{l}\text { Normal hsTnI } \\
\text { N (\%)/M(IQR) }\end{array}$ & $p$-Value & SMD \\
\hline$n$ & 375 & 126 & 249 & & \\
\hline Age (years) & $66(55.5-74)$ & $71.5(64.2-79)$ & $63(51-71)$ & $<0.001$ & 0.627 \\
\hline Age $\geq 65$ years old & $210(56)$ & $94(74.6)$ & $116(46.6)$ & $<0.001$ & 0.598 \\
\hline Male & $252(67.2)$ & $82(65.1)$ & $170(68.3)$ & 0.61 & 0.076 \\
\hline BMI $\left(\mathbf{k g} / \mathbf{m}^{2}\right)(n=337)$ & $28(25-32)$ & $29(26-34)$ & $28(25-31)$ & 0.04 & 0.035 \\
\hline eGFR $\left(\mathrm{mL} / \mathrm{min} / 1.73 \mathrm{~m}^{2}\right)$ on admission & $81(50-96)$ & $55.5(28-84)$ & $86(70-100)$ & $<0.001$ & 0.816 \\
\hline \multicolumn{6}{|l|}{ Cardiovascular risk factors } \\
\hline Hypertension $(n=374)$ & $221(58.9)$ & $96(76.2)$ & $125(50.2)$ & $<0.001$ & 0.575 \\
\hline Diabetes $(n=374)$ & $126(33.6)$ & $59(46.8)$ & $67(26.9)$ & $<0.001$ & 0.430 \\
\hline Dyslipidemia $(n=374)$ & $144(38.4)$ & $66(52.4)$ & $78(31.3)$ & $<0.001$ & 0.446 \\
\hline Smoking (history or current) $(n=330)$ & $80(21.3)$ & $30(23.8)$ & $50(20.1)$ & 0.36 & 0.121 \\
\hline Obesity $(n=347)$ & $134(35.7)$ & $51(40.5)$ & $83(33.3)$ & 0.20 & 0.122 \\
\hline \multicolumn{6}{|l|}{ Medical history } \\
\hline Heart disease $(n=374)$ & $65(17.3)$ & $31(24.6)$ & $34(13.7)$ & 0.007 & 0.305 \\
\hline Ischemic heart disease & $48(12.8)$ & $21(16.7)$ & $27(10.8)$ & 0.15 & 0.173 \\
\hline Chronic heart failure & $20(5.3)$ & $12(9.5)$ & $8(3.2)$ & 0.009 & 0.263 \\
\hline HFrEF & $13(3.5)$ & $7(5.5)$ & $6(2.4)$ & 0.20 & 0.478 \\
\hline Chronic kidney disease $(n=374)$ & 65 (17.3) & $32(25.4)$ & $33(13.2)$ & 0.003 & 0.316 \\
\hline Chronic respiratory disease $(n=374)$ & $45(12)$ & $16(12.7)$ & $29(11.6)$ & 0.73 & 0.305 \\
\hline Active cancer & $18(4.8)$ & $9(7.1)$ & $9(3.6)$ & 0.20 & 0.157 \\
\hline Cognitive impairment $(n=374)$ & $15(4)$ & $7(5.5)$ & $8(3.2)$ & 0.19 & 0.117 \\
\hline $\operatorname{VTE}(n=374)$ & $22(5.9)$ & $11(8.7)$ & $11(4.4)$ & 0.07 & 0.177 \\
\hline \multicolumn{6}{|l|}{ Admission treatment } \\
\hline Antithrombotic treatment & $121(32.3)$ & $55(43.7)$ & $66(26.5)$ & $<0.001$ & 0.387 \\
\hline Antiplatelet & $89(23.7)$ & $37(29.4)$ & $52(20.9)$ & 0.007 & 0.212 \\
\hline Anticoagulation & $41(10.9)$ & $23(18.3)$ & $18(7.2)$ & $<0.001$ & 0.351 \\
\hline \multicolumn{6}{|l|}{ Antihypertensive drugs } \\
\hline RASi & $150(40)$ & $63(50)$ & $87(34.9)$ & 0.006 & 0.308 \\
\hline Diuretics & $83(22.1)$ & $41(32.5)$ & $42(16.9)$ & $<0.001$ & 0.387 \\
\hline Beta-blockers & $103(27.5)$ & 47 (37.3) & $56(22.5)$ & $<0.001$ & 0.354 \\
\hline
\end{tabular}


Table 1. Cont.

\begin{tabular}{|c|c|c|c|c|c|}
\hline & $\begin{array}{l}\text { Overall Cohort } \\
\text { N (\%)/M(IQR) }\end{array}$ & $\begin{array}{l}\text { Elevated hsTnI } \\
\text { N (\%)/M(IQR) }\end{array}$ & $\begin{array}{l}\text { Normal hsTnI } \\
\text { N }(\%) / M(I Q R)\end{array}$ & $p$-Value & SMD \\
\hline Admission Low-dose chest CT ${ }^{*}$ & $320(85.3)$ & $102(81)$ & $218(87.6)$ & 1 & 0.009 \\
\hline abnormal & $314(98.1)$ & $100(98)$ & $245(98.4)$ & 1 & \\
\hline \multicolumn{6}{|l|}{ COVID-19 infection severity indicators } \\
\hline Oxygen therapy flow rate of $>5 \mathrm{~L} / \mathrm{min}$ & $231(67.9)$ & $82(77.4)$ & $149(63.7)$ & 0.01 & 0.304 \\
\hline ICU admission & $215(57.6)$ & 85 (67.5) & $130(52.6)$ & 0.008 & 0.307 \\
\hline Intubation & $205(54.8)$ & $84(66.7)$ & $121(48.8)$ & 0.001 & 0.353 \\
\hline HFNO therapy/NIV & $10(2.7)$ & $1(0.8)$ & $9(3.6)$ & 0.17 & \\
\hline CT scan extension $>25 \%(n=320)$ & $179(47.7)$ & $69(54.8)$ & $110(44.2)$ & 0.052 & 0.655 \\
\hline $\mathrm{CRP} \geq 100 \mathrm{mg} / \mathrm{L}(n=371)$ & $270(72)$ & $106(84.1)$ & $164(65.9)$ & $<0.001$ & 0.458 \\
\hline D-dimer count $\geq 3000 \mu \mathrm{g} / \mathrm{L}(n=292)$ & $170(45.3)$ & 77 (61.1) & $93(37.3)$ & $<0.001$ & 0.546 \\
\hline Lymphopenia $<1000 / \mu \mathrm{L}(n=371)$ & $284(75.7)$ & $104(82.5)$ & $180(72.2)$ & 0.026 & 0.276 \\
\hline \multicolumn{6}{|l|}{ In-hospital treatment } \\
\hline $\begin{array}{l}\text { Prophylactic/therapeutic } \\
\text { anticoagulation }\end{array}$ & $329(88.2)$ & $108(86.4)$ & $221(89.1)$ & 0.55 & \\
\hline Antibiotics & $331(88.3)$ & $109(86.5)$ & $222(89.2)$ & 0.49 & \\
\hline Antiviral ${ }^{\$}$ & $199(53.1)$ & $60(47.6)$ & $139(55.8)$ & 1 & \\
\hline \multicolumn{6}{|l|}{ In-hospital outcomes } \\
\hline Death & $90(24)$ & $60(47.6)$ & $30(12)$ & $<0.001$ & 0.844 \\
\hline Severe sepsis or septic shock & $98(27.2)$ & $47(37.9)$ & $51(21.6)$ & $<0.001$ & 0.389 \\
\hline Acute renal impairment & $136(36.3)$ & 77 (61.1) & 59 (23.7) & $<0.001$ & 0.851 \\
\hline VTE & $57(15.2)$ & $25(19.8)$ & $32(12.9)$ & 0.10 & 0.190 \\
\hline Stroke/TIA & $16(4.3)$ & $10(7.9)$ & $6(2.4)$ & 0.026 & 0.289 \\
\hline Hospital length of stay (days) & $15(8-29)$ & $16.5(8-35)$ & $15(8-27)$ & 0.26 & 0.277 \\
\hline
\end{tabular}

BMI: body mass index; CRP: C-reactive protein; CT: computer tomography; eGFR: estimated glomerular filtration rate; HFrEF: heart failure with reduced ejection fraction; hs-cTnI: high-sensitivity cardiac troponin I; HFNO: high-flow nasal oxygen; ICU: intensive care unit; IQR: interquartile range; M: median; N: number; NHF: nasal high flow; NIV: noninvasive ventilation; PCR: polymerase chain reaction; RASi: renin-angiotensin system inhibitor; SMD: standardized mean difference; VTE: venous thromboembolism; TIA: transient ischemic attack. ${ }^{*}$ number (frequencies) of low-dose CT scans performed in each group. \$ remdesivir, lopinavir/ritonavir, oseltamivir, interferon, hydroxychloroquine

\subsection{Troponin Elevation and in Hospital Outcomes}

A rise in troponin was associated with unfavorable outcomes such as severe sepsis, acute renal impairment, stroke, and death (Table 1, Figure 2).

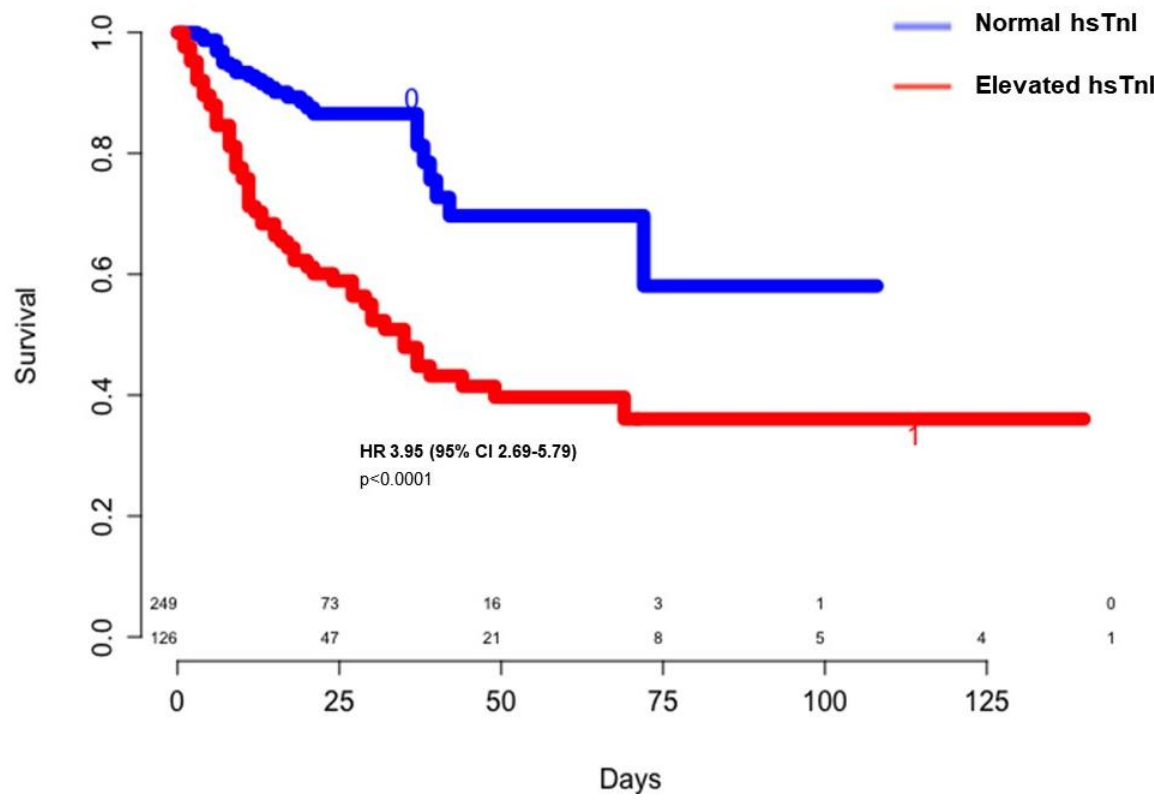

Figure 2. Crude survival rates according to high-sensitivity troponin levels. CI: confidence interval; HR: hazard ratio; hsTnI: high-sensitivity troponin I. 


\subsection{Mortality Predictors}

In the multivariate logistic regression analysis between survivors and nonsurvivors, including variables statistically significant in univariate analysis and deemed clinically pertinent, mortality was independently associated with an age of at least 65 years old (OR 3.17, 95\% CI 1.45-7.18), C-reactive protein (CRP) elevation of at least $100 \mathrm{mg} / \mathrm{L}$ (OR 3.62, 95\% CI 1.12-13.98), and hsTnI elevation (OR 3.12, 95\% CI 1.49-6.65) (Table 2).

Table 2. Univariate and multivariate analysis of baseline risk factors for death.

\begin{tabular}{lcccc}
\hline \multicolumn{1}{c}{ Risk Factor } & Unadjusted OR (95\% CI) & $p$-Value & Adjusted OR (95\% CI) & $p$-Value \\
\hline Age $\geq$ 65 years old & $5.55(3.12-10.47)$ & $<0.001$ & $3.17(1.45-7.18)$ & 0.004 \\
High blood pressure & $2.08(1.25-3.55)$ & 0.005 & $0.78(0.28-2.10)$ & 0.63 \\
Diabetes mellitus & $1.66(1.01-2.71)$ & 0.04 & $0.95(0.40-2.15)$ & 0.90 \\
Dyslipidemia & $1.60(0.98-2.59)$ & 0.054 & $0.54(0.22-1.25)$ & 0.16 \\
Tobacco consumption & $2.33(1.33-4.04)$ & 0.002 & $1.99(0.89-4.49)$ & 0.09 \\
Active Cancer & $5.52(2.10-15.47)$ & $<0.001$ & $2.80(0.67-11.65)$ & 0.15 \\
Chronic kidney disease & $3.07(1.74-5.41)$ & $<0.001$ & $2.30(0.84-6.41)$ & 0.10 \\
Ischemic heart disease & $2.39(1.25-4.50)$ & 0.007 & $1.02(0.30-3.36)$ & 0.96 \\
Chronic heart failure & $5.39(2.15-14.22)$ & $<0.001$ & $0.93(0.17-4.86)$ & 0.94 \\
Previous antithrombotic drug & $2.52(1.54-4.13)$ & $<0.001$ & $1.72(0.70-4.26)$ & 0.23 \\
Previous RASi & $1.61(1.00-2.60)$ & 0.049 & $1.40(0.55-3.61)$ & 0.47 \\
Lymphopenia & $2.35(1.25-4.77)$ & 0.011 & $3.05(0.90-14.35)$ & 0.10 \\
CRP $\geq 100$ mg/L (max) & $3.26(1.71-6.76)$ & $<0.001$ & $3.62(1.12-13.98)$ & 0.042 \\
D-dimer count (max) $\geq 3000 \mu \mathrm{pg} / \mathrm{L}$ & $2.52(1.42-4.62)$ & 0.002 & $1.55(0.70-3.55)$ & 0.28 \\
hsTroponin elevation & $6.63(3.98-11.25)$ & $<0.001$ & $3.12(1.49-6.65)$ & 0.003 \\
\hline
\end{tabular}

CI: confidence interval; CRP: C-reactive protein; hs: high-sensitivity; max: maximum; OR: odds ratio; RASi: renin-angiotensin system inhibitor.

After performing a sensitivity analysis on the entire cohort including patients without hsTnI measurement, age of at least 65 years old (OR 4.99, 95\% CI 2.69-9.25), active cancer (OR 2.52, 95\% CI 1.22-5.22), chronic kidney disease (OR 3.26, 95\% CI 1.85-5.76), CRP elevation of at least $100 \mathrm{mg} / \mathrm{L}$ (OR 2.34, 95\% CI 1.28-4.28), D-dimer elevation over $3000 \mu \mathrm{g} / \mathrm{L}$ (OR 1.95, 95\% CI 1.04-3.52), and hsTnI elevation (OR 3.84, 95\% CI 1.78-8.28) were significantly associated with death (Supplementary Table S1).

\section{Discussion}

\subsection{Troponin Elevation and in-Hospital Mortality}

In our study, a rise in hsTnI was identified in $34 \%$ of the cohort. Patients with hsTnI elevation had a higher prevalence of "cardiovascular burden," translating into a four-fold increased risk of death compared to normal hsTnI patients ( $47 \%$ vs. $12 \%)$. Furthermore, when performing a sensitivity analysis for missing values of hsTnI, a rise in troponin remained independently associated with death. Our findings are consistent with recently published data showing that cardiac biomarkers such as Tn, B-type natriuretic peptide, or D-dimer are commonly elevated in hospitalized COVID-19 patients [14]. However, a rise in Tn appears more accurate in predicting mortality compared to other biomarkers [14,17]. In a meta-analysis of 16 studies, Zou et al. found a pooled overall incidence of myocardial injury of $24.4 \%$ (542/2224) and a markedly increased all-cause mortality associated with a rise in Tn (72.6\% vs. 14.5\%) [12]. Furthermore, in the largest study published to date on Tn as a predictor of death including 6247 COVID-19 patients, Majure et al. observed significantly increased death rates in the case of Tn elevation compared to normal Tn levels (43\% vs. 13\%) [13].

Troponin assays differ among studies and details concerning cut-offs and manufacturers were not systematically reported in the literature [18]. Nonetheless, Tn remains an objective marker easily obtainable with an obvious prognosis impact. Indeed, Manocha et al. showed in a cohort of 446 patients that high Tn levels were a potent predictor of 30-day in-hospital mortality with an adjusted OR of 4.38 , allowing the authors to develop and validate a mortality score including age, hypoxia, and Tn elevation beyond the 75th percentile (HA2T2) to predict death [14]. 
TheACS-related rise in hsTnI in our study was modest $(2.3 \%)$ and is consistent with other retrospective studies reporting similar observations. Thus, Tn appears to have low specificity for ACS in the COVID-19 context, but myocardial injury could serve as a severity marker rather than being a cause of death per se $[13,14,19]$.

\subsection{Myocardial Injury in COVID-19: Definition and Potential Mechanisms}

Although most studies defined myocardial injury on the sole basis of Tn elevation, Giustino et al. performed an extensive study on echocardiographic abnormalities and found cardiac structural alterations in two-thirds of patients with enzymatic rise, which was strongly correlated to mortality [20]. The recent perception of COVID-19 as a systemic condition acting through multiple deleterious mechanisms, such as inflammation, endothelial dysfunction, prothrombotic state, and myocardial injury, could explain the severe course of the disease in some patients. The hypothetical mechanisms of myocardial injury include tissular hypoxia, cytokine storm, and direct viral myocardial lesions $[1,12,18]$.

\subsection{Limitations}

Given the retrospective nature of our study, only patients with a hsTnI measurement were included in the first analysis, which is susceptible to selection bias. However, performing a sensitivity analysis for missing values allowed us to confirm the initial results, adding robustness to our observation. Moreover, the use of two troponin measurement assays with different URLs did not allow a ROC-curve cut-off calculation. Nonetheless, high-sensitivity troponin tests are recognized as being more accurate than previous measurement techniques and are the recommended assay for assessing myocardial injury defined by an increase in hsTnI over the 99th percentile URL. As hsTnI URLs differ between assays, compiling data using hsTnI as a continuous variable could be biased. For this reason, we consider that using hsTnI elevation as a categorical variable was a practical alternative, palliating sex- and site-specific URLs and responding to guidelines' myocardial injury definition.

\subsection{Further Implications}

Although current ICU approaches have marginally changed between the first and second waves of the epidemics, potentially affecting the generalizability of our conclusions, there is still no available prognosis-changing antiviral treatment. Thus, given the considerable size of our population, we deem that our results could be pertinent for presently hospitalized patients as Tn is one of the earliest markers of end-organ dysfunction reflecting relative hypoxia [13,21]. As such, we advocate for Tn assessment to improve risk stratification and prompt more intensive surveillance with potential therapeutic consequences on enhancing tissular perfusion.

\section{Conclusions}

Elevated troponin measurement in COVID-19 hospitalized patients was independently correlated to in-hospital mortality and could serve as a predictor for short-term survival providing an indication for intensive clinical vigilance and potentially improving patients' triage.

Supplementary Materials: The following are available online at http://www.mdpi.com/2077-0383/9/12/4078/s1; Table S1: Univariate and multivariate analysis of baseline risk factors for death on sensitivity analysis with multiple imputation for missing hsTnI measurements $(n=772)$.

Author Contributions: Conceptualization, E.-M.C., N.D., V.S.-K. and D.S.; methodology, E.-M.C., N.D., A.D., W.Y. and D.S.; software, E.-M.C., L.J., H.L. and A.-S.F.; validation, E.-M.C., E.A., P.B., P.O., V.S.-K. and D.S.; formal analysis, E.-M.C. and F.S.; investigation, E.-M.C., L.J., H.L., J.T., C.M., H.M., P.B., P.O. and E.A.; resources, A.-S.F., E.-M.C. and D.S.; data curation, E.-M.C., L.J., H.L., J.T., A.D., W.Y. and A.-S.F.; writing-original draft preparation, E.-M.C. and D.S.; writing-review and editing, E.-M.C., E.A., F.S. and D.S.; visualization, E.-M.C. and D.S.; supervision, D.S.; project administration, E.-M.C. and D.S. All authors have read and agreed to the published version of the manuscript.

Funding: This research received no external funding. 
Acknowledgments: The authors would like to thank Veronique Kemmel for her biochemistry expertise in the field of high-sensitivity troponin I measurement.

Conflicts of Interest: The authors declare no conflict of interest.

\section{References}

1. Li, B.; Yang, J.; Zhao, F.; Zhi, L.; Wang, X.; Liu, L.; Bi, Z.; Zhao, Y. Prevalence and impact of cardiovascular metabolic diseases on COVID-19 in China. Clin. Res. Cardiol. 2020, 109, 531-538. [CrossRef]

2. Wang, Q.; Zhang, Y.; Wu, L.; Niu, S.; Song, C.; Zhang, Z.; Lu, G.; Qiao, C.; Hu, Y.; Yuen, K.-Y.; et al. Structural and Functional Basis of SARS-CoV-2 Entry by Using Human ACE2. Cell 2020, 181, 894-904.e9. [CrossRef] [PubMed]

3. Yan, R.; Zhang, Y.; Li, Y.; Xia, L.; Guo, Y.; Zhou, Q. Structural basis for the recognition of SARS-CoV-2 by full-length human ACE2. Science 2020, 367, 1444-1448. [CrossRef]

4. Samavati, L.; Uhal, B.D. ACE2, Much More Than Just a Receptor for SARS-COV-2. Front. Cell. Infect. Microbiol. 2020, 10. [CrossRef]

5. Chen, L.; Li, X.; Chen, M.; Feng, Y.; Xiong, C. The ACE2 expression in human heart indicates new potential mechanism of heart injury among patients infected with SARS-CoV-2. Cardiovasc. Res. 2020, 116, 1097-1100. [CrossRef] [PubMed]

6. Varga, Z.; Flammer, A.J.; Steiger, P.; Haberecker, M.; Andermatt, R.; Zinkernagel, A.S.; Mehra, M.R.; Schuepbach, R.A.; Ruschitzka, F.; Moch, H. Endothelial cell infection and endotheliitis in COVID-19. Lancet 2020, 395, 1417-1418. [CrossRef]

7. Januzzi, J.L. Troponin and BNP Use in COVID-19. 2020. Available online: https://www.acc.org/latestincardiology/articles/2020/03/18/15/25/troponin-and-bnp-use-in-covid19 (accessed on 8 November 2020).

8. Wang, D.; Hu, B.; Hu, C.; Zhu, F.; Liu, X.; Zhang, J.; Wang, B.; Xiang, H.; Cheng, Z.; Xiong, Y.; et al. Clinical Characteristics of 138 Hospitalized Patients with 2019 Novel Coronavirus-Infected Pneumonia in Wuhan, China. JAMA 2020, 323, 1061. [CrossRef]

9. Guo, T.; Fan, Y.; Chen, M.; Wu, X.; Zhang, L.; He, T.; Wang, H.; Wan, J.; Wang, X.; Lu, Z. Cardiovascular Implications of Fatal Outcomes of Patients with Coronavirus Disease 2019 (COVID-19). JAMA Cardiol. 2020, 5, 811. [CrossRef]

10. Shi, S.; Qin, M.; Shen, B.; Cai, Y.; Liu, T.; Yang, F.; Gong, W.; Liu, X.; Liang, J.; Zhao, Q.; et al. Association of Cardiac Injury with Mortality in Hospitalized Patients with COVID-19 in Wuhan, China. JAMA Cardiol. 2020, 5, 802-810. [CrossRef]

11. Lala, A.; Johnson, K.W.; Januzzi, J.L.; Russak, A.J.; Paranjpe, I.; Richter, F.; Zhao, S.; Somani, S.; Van Vleck, T.; Vaid, A.; et al. Prevalence and Impact of Myocardial Injury in Patients Hospitalized with COVID-19 Infection. J. Am. Coll. Cardiol. 2020, 76, 533-546. [CrossRef] [PubMed]

12. Zou, F.; Qian, Z.; Wang, Y.; Zhao, Y.; Bai, J. Cardiac Injury and COVID-19: A Systematic Review and Meta-analysis. CJC Open 2020, 2, 386-394. [CrossRef] [PubMed]

13. Majure, D.T.; Gruberg, L.; Saba, S.G.; Kvasnovsky, C.; Hirsch, J.S.; Jauhar, R. Usefulness of Elevated Troponin to Predict Death in Patients with COVID-19 and Myocardial Injury. Am. J. Cardiol. 2020. [CrossRef] [PubMed]

14. Manocha, K.K.; Kirzner, J.; Ying, X.; Yeo, I.; Peltzer, B.; Ang, B.; Li, H.A.; Lerman, B.B.; Safford, M.M.; Goyal, P.; et al. Troponin and Other Biomarker Levels and Outcomes Among Patients Hospitalized with COVID-19: Derivation and Validation of the HA 2 T 2 COVID-19 Mortality Risk Score. J. Am. Hear. Assoc. 2020, e018477. [CrossRef] [PubMed]

15. Pedersen, A.B.; Mikkelsen, E.M.; Cronin-Fenton, D.P.; Kristensen, N.R.; Pham, T.M.; Pedersen, L.; Petersen, I. Missing data and multiple imputation in clinical epidemiological research. Clin. Epidemiol. 2017, 9, 157-166. [CrossRef] [PubMed]

16. van Buuren, S.; Groothuis-Oudshoorn, K. mice: Multivariate Imputation by Chained Equations in R. J. Stat. Softw. 2011, 45,1-68. [CrossRef]

17. De Almeida, G.L.G.; Braga, F.; Jorge, J.K.; Nobre, G.F.; Kalichsztein, M.; Faria, P.D.M.P.D.; Bussade, B.; Penna, G.L.; Alves, V.O.; Pereira, M.A.; et al. Valor Prognóstico da Troponina T e do Peptídeo Natriurético Tipo B em Pacientes Internados por COVID-19. Arq. Bras. Cardiol. 2020, 115, 660-666. [CrossRef] [PubMed]

18. Kavsak, P.A.; Hammarsten, O.; Worster, A.; Smith, S.W.; Apple, F.S. OUP accepted manuscript. Clin. Chem. 2020. [CrossRef] 
19. Bangalore, S.; Sharma, A.; Slotwiner, A.; Yatskar, L.; Harari, R.; Shah, B.; Ibrahim, H.; Friedman, G.H.; Thompson, C.; Alviar, C.L.; et al. ST-Segment Elevation in Patients with Covid-19-A Case Series. N. Engl. J. Med. 2020, 382, 2478-2480. [CrossRef] [PubMed]

20. Giustino, G.; Croft, L.B.; Stefanini, G.G.; Bragato, R.; Silbiger, J.J.; Vicenzi, M.; Danilov, T.; Kukar, N.; Shaban, N.; Kini, A.; et al. Characterization of Myocardial Injury in Patients with COVID-19. J. Am. Coll. Cardiol. 2020, 76, 2043-2055. [CrossRef] [PubMed]

21. Chapman, A.R.; Bularga, A.; Mills, N.L. High-Sensitivity Cardiac Troponin Can Be an Ally in the Fight against COVID-19. Circulation 2020, 141, 1733-1735. [CrossRef] [PubMed]

Publisher's Note: MDPI stays neutral with regard to jurisdictional claims in published maps and institutional affiliations.

(C) 2020 by the authors. Licensee MDPI, Basel, Switzerland. This article is an open access article distributed under the terms and conditions of the Creative Commons Attribution (CC BY) license (http://creativecommons.org/licenses/by/4.0/). 